\title{
Correspondence
}

\section{New feeder for infants with cleft palate}

Sir,

Early feeding difficulties in infants with a cleft palate are well documented ${ }^{1}$ as is poor mother-infant bonding. ${ }^{2}$ For many years these infants have been fed with various makeshift modifications of a teat. Over the last year we have been using a new feeder specifically designed to overcome many of the problems of feeding such infants.

The new 'Haberman' feeder consists of four components (see figure). The flanged cylinder (solid, food grade silicone) has one end formed to accept a mushroom valve through which the milk enters the teat; this provides a non-return passage for milk from a conventional feeding bottle. A lateral channel is incorporated in the posterior aspect of the flange to allow air to enter the bottle, preventing a vacuum being formed in it. The teat is made from tear resistant food grade silicone. It is soft, malleable, and transparent and fits over the cylinder to form a liquid and airtight seal. The mouthpiece has a slit valve opening with markings on the teat body to indicate the position of the slit opening relative to the infant's mouth; this controls the flow of milk to the infant's mouth. Sucking by the infant compresses the teat alone so that it is not wasted on compressing air inside the bottle. The feeder is attached to the bottle by a collar.

We have recently used the new feeder with six infants: two had bilateral clefts and the remaining four had unilateral clefts. Their gestational ages ranged from 36 to 42 weeks with a mean weight of $2.8 \mathrm{~kg}$ (range $1.9 \mathrm{~kg}-3.2$ $\mathrm{kg}$ ). Three infants were initially fed with conventional teats while the remaining three used the Haberman feeder from birth. It was found that the infants fed faster and more

\section{Haberman feeder}

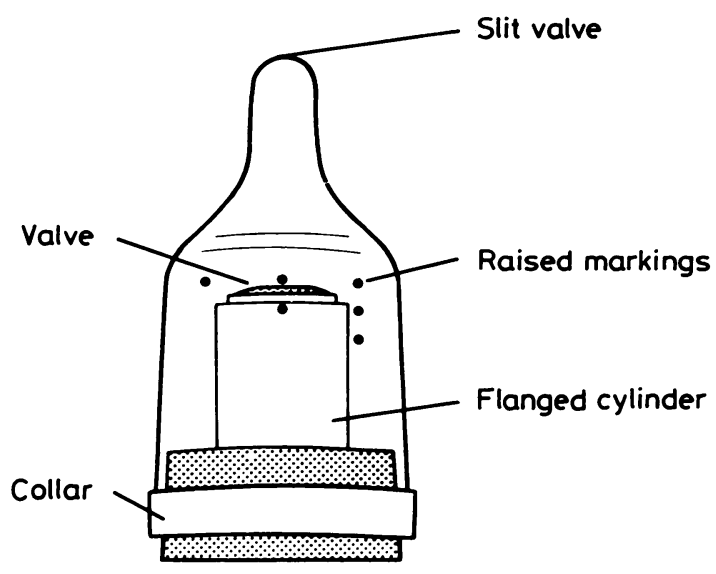

easily with the new feeder. There was less aerography and consequently less vomiting. The weight gain was satisfactory throughout. The parents had no complaints while using the feeder and in those where a conventional teat was used first, the Haberman feeder was preferred.

We believe that the Haberman feeder is a significant advance in the feeding of infants with cleft palates and should be the preferred feeder rather than conventional means. A further long term study is underway.

The feeder is manufactured by Camberley Rubber Mouldings Ltd, Farnborough, Hampshire.

\section{References}

${ }^{1}$ Kelly E. Feeding cleft palate babies. Cleft Palate J 1971; 8: 61-4.

2 Tisza VB, Gompertz E. The parents' reaction to the birth and early care of children with cleft palate. Pediatrics 1962; 30: 86-90.

A N Campbell and M J Tremouth, Departments of Paediatrics and Orthodontics, Royal Preston Hospital, Preston, Lancashire PR2 4HT

\section{Radionuclide scans in reflux nephropathy}

Sir,

The excellent article by Professor White on the management of urinary tract infection mentions radionuclide scans only briefly. ${ }^{1}$ A technetium dimercaptosuccinate (DMSA) scan is an indispensible tool in the assessment of reflux nephropathy. ${ }^{2}$ Ultrasound has an advantage over the intravenous urogram (IVU) in a young child because the kidneys are accessible to a high frequency high resolution transducer and the delineation of renal anatomy is usually better than on an IVU with poor contrast excretion and interfering bowel gas shadows. Certainly in children under the age of 3 years ultrasound should be the first investigation, followed by a DMSA scan as the second investigation, once an obstruction has been excluded.

In a consecutive series of 25 infants studied by DMSA scans within three weeks of their first febrile urinary tract infection, 15 kidneys in 10 infants were found to have focal parenchymal defects suggestive of upper tract involvement. ${ }^{3}$ Cystograms in these 10 infants subsequently showed ipsilateral reflux to 10 of the abnormal kidneys, five kidneys were drained by non-refluxing ureters and no contralateral reflux was observed to kidneys which were normal on DMSA scanning. These observations have subsequently been extended and we have found that the DMSA scan exceeds both the IVU and ultrasound in its ability to detect the kidney at risk from reflux nephropathy (publication in preparation). Merrick et al compared the 